\title{
IMPLEMENTATION OF A LOW-MACH NUMBER MODIFICATION FOR HIGH-ORDER FINITE-VOLUME SCHEMES FOR ARBITRARY HYBRID UNSTRUCTURED MESHES
}

\author{
N.Simmonds ${ }^{1}$, P.Tsoutsanis ${ }^{2}$, and A.Gaylard ${ }^{2}$ \\ 1,2 Fluid Mechanics and Computational Sciences, Cranfield University, \\ Cranfield MK43 OAL, United Kingdom \\ e-mail: n.simmonds@cranfield.ac.uk, panagiotis.tsoutsanis@cranfield.ac.uk \\ ${ }^{3}$ Jaguar Land Rover \\ Banbury Road, Gaydon, Warwick, CV35 ORR \\ e-mail: agaylar1@jaguarlandrover.com
}

Keywords: Instructions, ECCOMAS Congress, Computational Methods, Engineering Sciences, Proceedings.

\begin{abstract}
An implementation of a novel low-mach number treatment for high-order finite-volume schemes using arbitrary hybrid unstructured meshes is presented in this paper. Low-Mach order modifications for Godunov type finite-volume schemes have been implemented successfully for structured and unstructured meshes, however the methods break down for hybrid mesh topologies containing multiple element types. The modification is applied to the UCNS3D finite-volume framework for compressible flow configurations, which have been shown as very capable of handling any type of grid topology. The numerical methods under consideration are the Monotonic UpstreamCentered Scheme for Conservation Laws (MUSCL) and the Weighted Essentially NonOscillatory (WENO) schemes for two-dimensional mixed-element type unstructured meshes. In the present study the HLLC Approximate Riemann Solver is used with an explicit TVD Runge-Kutta 3rd-order method due to its excellent scalability. These schemes (up to 5th-order) are applied to well established two-dimensional and three-dimensional test cases. The challenges that occur when applying these methods to low-mach flow configurations is thoroughly analysed and possible improvements and further test cases are suggested.
\end{abstract}




\section{Introduction}

Obtaining accurate solutions in an efficient manner for a wide-range of flow problems where the compressible Navier-Stokes are used is the main goal of the majority of the numerical methods and schemes developed in this context. The most challenging part of the development of all the high-resolution numerical schemes used is that they should be adaptive. Adaptive in the sense of identifying regions of sharp-gradients often encountered in compressible flows, and preventing or eliminating any spurious oscillations that can occur and contaminate the solution; but at the same time they should be adaptive and achieve high-order of accuracy in smooth regions of the flow. However there is a delicate balance between the two requirements and is dependent upon the spatial discretisation method, the shock-capturing algorithms, the grid types, the Riemann solvers, the time-stepping algorithms and the quadrature rules used for integration to name a few.

The first generation numerical methods for unstructured grids exhibited lower accuracy and were computationally more demanding than structured grids. However, the numerical methods for unstructured grids have matured and numerous elegant approaches $[1,2,3,4,5,6,7,8,9,10,11,12,13]$ and algorithms have been developed in the finite volume framework for a wide range of applications for Computational Fluid Dynamics(CFD). Other state-of-the-art approaches are also available, such as the Discontinuous Galerkin (DG) [14, 15, 2, 16, 11, 17], and Spectral Finite-Volume (SFV) methods [18, 19, 20, 12, 21, 22] that have been successfully applied for CFD applications. For the finite volume framework the first class of high-resolution methods developed for unstructured grids included the ENO type schemes [23, 24], followed by the WENO type schemes [25, 26, 27, 28]. In the WENO case, the high-order accuracy was achieved by non-linearly combining a series of high-order reconstruction polynomials arising from a series of reconstruction stencils. Recently, a class of WENO type methods [9, 8] has been successfully extended to hybrid unstructured meshes with various geometrical shapes such as tetrahedrals, hexahedrals, prisms, and pyramids. The schemes can achieve the very high order of spatial accuracy across interfaces between cells of different types, and at the same time essentially non-oscillatory profiles are produced for discontinuous solutions. This gives greater flexibility to handle complex geometrical shapes in an efficient and accurate manner.

For the majority of the finite-volume numerical methods for compressible flows their dissipation characteristics are proportional to the speed of sound, therefore the low Mach number features are damped by the numerical scheme as noted by [29]. This is particular important at regions of the flow where the Mach number is low such as close to the wall at the boundary layer, and at vortices arising from shear layers.

In the present paper we present a modification to the output of the reconstruction, or the input of the Riemann solver in order to remove the Mach number dependence, and improve the resolution at low-Mach regions of the flow. It has to be noted that the 
subject modification is different in nature from a preconditioning step used for changing the flow variables, since our main motivation is not to relax the restriction in terms of the time-step size, but rather to increase the resolution at the low-Mach regions. The original modification proposed by [29] is not directly transferable to any grid-type since different mesh elements have different dissipation characteristics, therefore a unified modification is implemented that is suitable for all element types and through the computational results presented we demonstrate the difference between the original modification, and the revised one. The nice feature of this modification is that is quite simple to implement in any compressible code, for any numerical scheme that uses a Riemann solver and the additional computational expense is negligible.

\section{Governing Equations}

The compressible inviscid Navier-Stokes equations are solved; writen in compact form as:

$$
\frac{\partial \mathbf{U}(\mathbf{x}, t)}{\partial t}+\nabla \mathbf{F}_{\mathbf{c}}(\mathbf{U})=0
$$

where $\mathbf{U}$ is the vector of the conserved mean flow variables; $\mathbf{F}_{\mathbf{c}}$, is the inviscid flux vector given by:

$$
\begin{aligned}
& \mathbf{U}=[\rho, \rho u, \rho v, \rho w, \rho E]^{T}, \\
& F_{c}^{\mathbf{x}}=\left[\rho u, \rho u^{2}+p, \rho u v, \rho u w, u(E+p)\right]^{T} \\
& F_{c}^{\mathbf{y}}=\left[\rho v, \rho u v, \rho v^{2}+p, \rho v w, v(E+p)\right]^{T} \\
& F_{c}^{\mathbf{z}}=\left[\rho w, \rho u w, \rho v w, \rho w^{2}+p, w(E+p)\right]^{T}
\end{aligned}
$$

In the above equations, $\rho$ is the density; $u, v, w$ are the velocity components in $x, y$ and $z$ Cartesian directions, respectively. Calorically perfect gas is assumed where the total energy per unit mass is computed according to the equation of state as $E=p /(\gamma-1)+$ $(1 / 2) \rho\left(u^{2}+v^{2}+w^{2}\right)$, where $p$ is the pressure, the ratio of specific heats is set as $\gamma=1.4$ for air at normal atmoshperic conditions.

\section{Numerical Framework}

The discretization in a domain $\Omega$ is achieved by combining conforming arbitrary shaped elements of volume $\left|V_{i}\right|$. Integrating Eq. (1) over a mesh element using the finite-volume formulation the following ordinary differential equation is obtained

$$
\frac{d \mathbf{U}_{i}}{d t}=-\frac{1}{\left|V_{i}\right|} \sum_{l=1}^{N_{f}} \sum_{\alpha=1}^{N_{q p}} \mathbf{F}_{c}^{\mathbf{n}, \mathbf{l}}\left(\mathbf{U}\left(\mathbf{x}_{\alpha}, t\right)\right) \omega_{\alpha}\left|A_{l}\right|
$$


where $\mathbf{U}_{i}$ is the volume averaged conserved variables in a volume element, $N_{f}$ is the number of faces per element, $N_{q p}$ is the number of quadrature points used for approximating the surface integrals. $\left|A_{l}\right|$ is the surface area of the corresponding face, and $\alpha$ corresponds to different Gaussian integration points $\mathbf{x}_{\alpha}$ and weights $\omega_{\alpha}$ over the face. The weight and distribution of the quadrature points depend upon the Gaussian rule order, higher integration rule will result in enhanced intercell flux approximation. The convective flux tensors are defined as:

$$
\mathbf{F}_{c}^{\mathbf{n}, \mathbf{l}}=F_{c}^{\mathbf{x}} n_{x}+F_{c}^{\mathbf{y}} n_{y}+F_{c}^{\mathbf{z}} n_{z}
$$

where $n_{x}, n_{y}$ and $n_{z}$ are the Cartesian components of the normal vector on the intercell surface. The intercell fluxes are computed based on volume averaged quantities which are obtained by an interpolation technique. The solution is obtained by a polynomial reconstruction from cell-averaged data. The following sections describe the methodology adopted for space and time discretization.

\subsection{Spatial Discretization}

The spatial discretisation is based on the approach of [8, 9], which is suitable for unstructured meshes with various types of element shapes in 2D and 3D, and it has been previously used successfully for laminar, transitional and turbulent flows [13, 30, 31, 32, 33]. Therefore, only the key characteristics of this approach are going to be described in this paper. The main objective of the reconstruction process is to build a high-order polynomial $p_{i}(x, y, z)$ of arbitrary order $r$, for each considered element $V_{i}$ that has the same average as a general quantity $\mathbf{U}_{i}$. This can be formulated as

$$
\mathbf{U}_{i}=\frac{1}{\left|V_{i}\right|} \int_{V_{i}} \mathbf{U}(x, y, z) d V=\frac{1}{\left|V_{i}\right|} \int_{V_{i}} p_{i}(x, y, z) d V .
$$

The reconstruction is carried out in a transformed system of coordinates in order to minimize scaling effects that appear in stencils consisting of elements of different size as well as to improve the condition number of the system of equations [13, 8]. The transformation is achieved by decomposing each element into tetrahedrals.

The reconstruction polynomial at the transformed cell $V_{i}^{\prime}$ is expanded over local polynomial basis functions labeled as $\phi_{k}(\xi, \eta, \zeta)$, which are given by

$$
p(\xi, \eta, \zeta)=\sum_{k=0}^{K} a_{k} \phi_{k}(\xi, \eta, \zeta)=\mathbf{U}_{0}+\sum_{k=1}^{K} a_{k} \phi_{k}(\xi, \eta, \zeta)
$$

where $\xi, \eta, \zeta$ are the coordinates in the reference system, $a_{k}$ are the degrees of freedom and the upper index in the summation of expansion $K$ is related to the order of the polynomial $r$ by $K=\frac{1}{6}(r+1)(r+2)(r+3)-1$ for 3D and $K=\frac{1}{3}(r+1)(r+2)-1$ for 
2D . For computing the degrees of freedom $a_{k}$, a minimum of $K$ cells is required in the stencil in addition to the target cell. Using the minimum possible number of cells in the stencil, $M \equiv K$, may produce ill-conditioned systems, hence using $M=2 \cdot K$ improves the robustness of the method as described in [8, 34]. A linear least-square method is adopted to enable the system of equations of 6 for the unknown degrees of freedom $a_{k}$. The final form of the linear system is solve with the QR decomposition algorithm. Discontinuous solutions are often encounter in external aerodynamics, thus limiting functions are essential for maintaning numerical stability and suppress any spurious oscillations. Two approaches are assessed in this work the MUSCL and the WENO schemes.

\subsubsection{MUSCL}

The MUSCL scheme is employed in this work is partially based on the Barth and Jespersen slope limiter. The design of the scheme requires the minimum and maximum values in the stencil's neighborhood, i.e., $U_{i}^{\min }=\min \left(U_{i}, U_{l}\right)$ and $U_{i}^{\max }=\max \left(U_{i}, U_{l}\right)$, where $l=1, . . L ; L$ is the total number neighbors of element $i$. The gradient of a general quantity is defined as $\nabla U_{i}$ and is an approximation of the solution gradient inside the element $i$. The gradient is computed during the reconstruction process by incorporating information from the entire central stencil. The scheme can be written as

$$
U_{i \alpha}=U_{i}^{c}+\phi_{i} \nabla U_{i} \cdot \mathbf{x}_{\alpha}
$$

where $U_{i}^{c}$ is the value for the general quantity at the element centroid and $\mathrm{x}_{\alpha}$ are the coordinates of the quadrature point. The limiter seeks the minimum value of the slope limiter for all the quadrature points that satisfy the following conditions

$$
\phi_{i}=\min \left(\bar{\phi}_{i, m_{1}}, \bar{\phi}_{i, m_{2}}, \ldots \bar{\phi}_{i, M}\right)
$$

Then, the limiting function is applied, composed by three different states according to the difference of the reconstructed value at the quadrature points of the considered element $U_{(i, \alpha)}$ and each of its neighbors $U_{l}$, yielding

$$
\bar{\phi}_{i, \alpha}=\left\{\begin{array}{cl}
\min \left(1, \frac{U_{i}^{\max }-U_{i, \alpha}}{U_{l}-U_{i, \alpha}}\right), & \text { if } U_{l}-U_{i, \alpha}>0 \\
\min \left(1, \frac{U_{i}^{\text {min }}-U_{i, \alpha}}{U_{l}-U_{i, \alpha}}\right), & \text { if } U_{l}-U_{i, \alpha}<0 \\
1, & \text { if } U_{l}-U_{i, \alpha}=0
\end{array}\right.
$$

\subsubsection{WENO}

WENO schemes use a non-linear combination of various reconstruction polynomials, where each polynomial is weighted according to the smoothness of its solution. The 
polynomials are given by

$$
p_{i}^{\text {weno }}=\sum_{m=1}^{m_{s}} \omega_{m} p_{m}(\xi, \eta, \zeta)
$$

where $m_{s}$ is the total number of WENO stencils. Substituting back to Eq. (6) for $p_{m}(\xi, \eta, \zeta)$, we obtain the following expression

$$
p_{m}(\xi, \eta, \zeta)=\sum_{m=0}^{K} a_{k}^{(m)} \phi_{k}(\xi, \eta, \zeta)
$$

Using the condition that the sum of all weights is unity, yields

$$
\begin{aligned}
p_{i}^{\text {weno }} & =\mathbf{U}_{0}+\sum_{k=1}^{K}\left(\sum_{m=0}^{m_{s}} \omega_{m} a_{k}^{m}\right) \phi_{k}(\xi, \eta, \zeta) \\
& \equiv \mathbf{U}_{0}+\sum_{k=1}^{K} \tilde{a}_{k} \phi_{k}(\xi, \eta, \zeta)
\end{aligned}
$$

where $\tilde{a}_{k}$ are the reconstructed degrees of freedom; and the non-linear weight $\omega_{m}$ is defined by [13, 8, 34]:

$$
\omega_{m}=\frac{\tilde{\omega}_{m}}{\sum_{m=0}^{m_{s}} \tilde{\omega}_{m}} \quad \text { where } \quad \tilde{\omega}_{m}=\frac{\lambda_{m}}{\left(\epsilon+\mathcal{I}_{m}\right)^{b}}
$$

The smoothness indicator is given by

$$
\mathcal{I}_{m}=\sum_{1 \leq|\beta| \leq r} \int_{\tilde{E}_{i}}\left(\mathcal{D}^{\beta} p_{m}(\xi, \eta, \zeta)\right)^{2}(d \xi, d \eta, d \zeta)
$$

where $\beta$ is a multi-index, $r$ is the polynomial's order, $\lambda_{m}$ is the linear weight, and $D$ is the derivative operator. The smoothness indicator is a quadratic function of the degrees of freedom $\left(a_{k}^{m}\right)$ and can be expressed as a universal mesh-independent oscillation indicator matrix as defined in [34]. The WENO reconstruction can be carried out in terms of conserved or characteristic variables. In this work, the conserved variables have been employed to assess the low-Mach number reconstruction modification. The various reconstruction polynomials arise from different sets of stencils that satisfy some geometrical conditions. The reader is referred to [9, 8] for the definition of geometrical sectors., and references therein, for a detailed explanation of the different set of geometrical conditions and WENO characteristic reconstruction. 


\subsection{Low-Mach Number Modification}

Our low-Mach number modification follows the previous work of [29], where the left and right state of the reconstructed velocities is modified in a linear way with respect to Mach number, so when the Mach number approaced zero the arithmetic mean of them is used in the following manner:

$$
\begin{aligned}
& u_{L}^{*}=\frac{(1+z) u_{L}+(1-z) u_{R}}{2} \\
& u_{R}^{*}=\frac{(1+z) u_{R}+(1-z) u_{L}}{2} \\
& z=\min \left(1, \max \left(M_{L}, M_{R}\right)\right)
\end{aligned}
$$

The Mach numbers are calculated based on the velocity magnitude of all the velocity components independent of the normal direction in which the flux is computed. However we investigate two approaches one where all the component of the reconstructed velocities are modified as the original implementation [29], and a second one where only the velocity components normal to the face are modified, based on the findings of [29] where it was highlighted that the tangential velocities require more numerical dissipation than the normal components.

\subsection{Fluxes Approximation}

Having reconstructed the cell-averaged solutions, the intercell fluxes can be evaluated. The Riemann problem is solved with the approximate Harten-Lax-van LeerContact (HLLC) solver of Toro [35]. The HLLC solver is also employed for the convective part of the turbulence transport equation. The HLLC flux function is given by

$$
\hat{\mathrm{F}}^{\text {HLLC }} \begin{cases}\hat{F}^{-}, & \text {if } 0 \leq S^{-}, \\ \hat{F}^{*-}=\hat{F}^{-}+S^{-}\left(\hat{W}^{*-}-\hat{W}^{-}\right), & \text {if } S^{-} \leq 0 \leq S^{*}, \\ \hat{F}^{*+}=\hat{F}^{+}+S^{+}\left(\hat{W}^{*+}-\hat{W}^{+}\right), & \text {if } S^{*} \leq 0 \leq S^{+}, \\ \hat{F}^{+}, & \text {if } 0 \geq S^{+},\end{cases}
$$

where

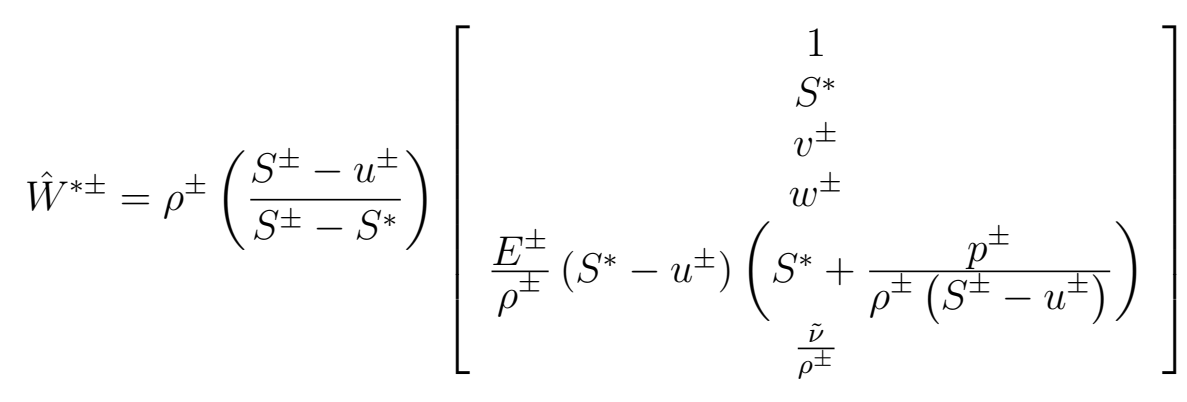


$\hat{W}^{* \pm}$ is computed either for the considered element "-", or for its neighbour "+" and the wave speeds are estimated according to the sign.

\subsection{Temporal discretisation}

Having constructed the numerical fluxes $\mathbf{F}_{n, j}$ as expressed in the semi-discrete conservative formulation, the next step involves the advancement of the solution in time. The explicit SSP Runge-Kutta $3^{\text {rd }}$-order method [36] has been employed for the time integration

$$
\left.\begin{array}{c}
\mathbf{U}_{\mathbf{i}}^{1}=\mathbf{U}_{\mathbf{i}}^{n}+\Delta t \cdot \mathbf{R}_{i}\left(\mathbf{U}_{\mathbf{i}}{ }^{n}\right) \\
\mathbf{U}_{\mathbf{i}}{ }^{2}=\frac{3}{4} \mathbf{U}_{\mathbf{i}}^{n}+\frac{1}{4} \mathbf{U}_{\mathbf{i}}{ }^{1}+\frac{\Delta t}{4} \cdot \mathbf{R}_{i}\left(\mathbf{U}^{1}\right) \\
\mathbf{U}_{\mathbf{i}}^{n+1}=\frac{1}{3} \mathbf{U}_{\mathbf{i}}^{n}+\frac{2}{3} \mathbf{U}_{\mathbf{i}}{ }^{2}+\frac{2 \Delta t}{3} \cdot \mathbf{R}_{i}\left(\mathbf{U}_{\mathbf{i}}{ }^{2}\right)
\end{array}\right\}
$$

with $\mathbf{R}_{i}$ being the residual.

The time step $\Delta t$ is computed as follows

$$
\Delta t=K \min _{i} \frac{h_{i}}{S_{i} \cdot V_{i}},
$$

where $h_{i}$ is the radius of the inscribed sphere of each cell $i$ and $V_{i}$ its corresponding volume, $K \leq 1 / 3$ is the CFL number for unsplit finite-volume schemes [35], and $S_{i}$ is the maximum propagation speed in each cell $i$ given by

$$
S_{i}=s p_{x} \cdot n_{x}+s p_{y} \cdot n_{y}+s p_{z} \cdot n_{z}
$$

where

$$
s p_{x}=|u+a|, \quad, s p_{y}=|v+a|, \quad s p_{x}=|w+a|,
$$

with $\mathbf{n}=\left(n_{x}, n_{y}, n_{z}\right)$ being the outward unit normal vector and $a$ is the speed of sound.

\section{Results}

This section presents the results obtained for a variety of cases to illustrate the influence of the low-mach modification compared to the previously implemented version.

\subsection{D Vortex Evolution}

The two-dimensional vortex evolution problem is an important case to consider for convergence and accuracy. This is a well used problem for low-Mach testing and illustrating the power of high order methods, [37]. 
N.Simmonds, P.Tsoutsanis, and A.Gaylard

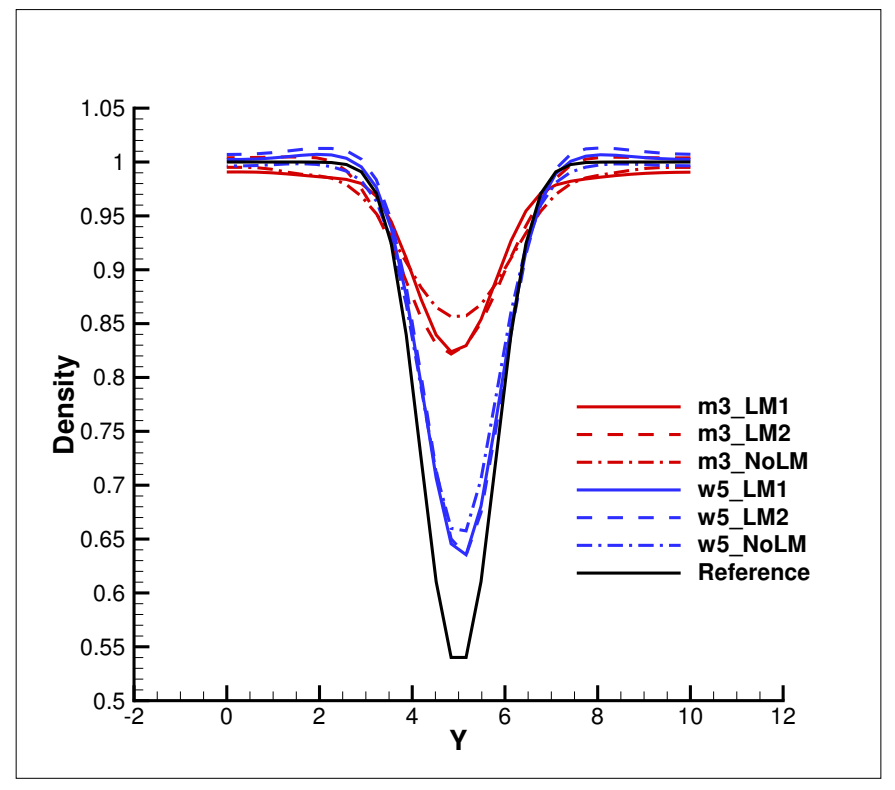

Figure 1: Density plots using a MUSCL 3rd and WENO 5th order schemes for the the vortex evolution flow problem on a quadrilatel mesh at $t=2 \mathrm{~s}$

We examine a square domain of area $\Omega=100,[0,10] x[0,10]$, with periodic boundary conditions. The mean flow of the problem is $\rho=1, P=1, u=1, v=1$, therefore there is a diagonal flow through the domain moving to the top right corner. The flow is pertubated by an isotropic vortex in the u and v fields with a temperature $T=\frac{P}{\rho}$ and no pertubation in the entropy $S=\frac{P}{\rho^{\gamma}}$, i.e. $\delta S=0$. The pertubations are given by

$$
\begin{gathered}
(\delta u, \delta v)=\frac{\epsilon}{2 \pi} e^{0.5\left(1-r^{2}\right)}(5-y, x-5) \\
\delta T=-\frac{(\gamma-1) \epsilon^{2}}{8 \gamma \pi^{2}} e^{1-r^{2}} \\
r^{2}=(x-5)^{2}+(y-5)^{2}
\end{gathered}
$$

The solution is computed at $t=2 s$ for both low-mach corrections using a 3rd order MUSCL scheme and a Fth order WENO scheme. These are compared to the initial solution $t=0 \mathrm{~s}$ on the same grid of quadrilateral elements with $32 \times 32$ elements. From Fig. 11, it can immediately been seen that the higher order WENO 5 scheme reduces the noticeable dissipation of the MUSCL scheme, with and without the low-mach correcions. The influence of the low-mach corrections on the 3rd order MUSCL simulations is higher and a clear difference can be seen in its absence resulting in less dissipation. It is noted that for this particular test case there is not a large difference between the two modifications.

9 


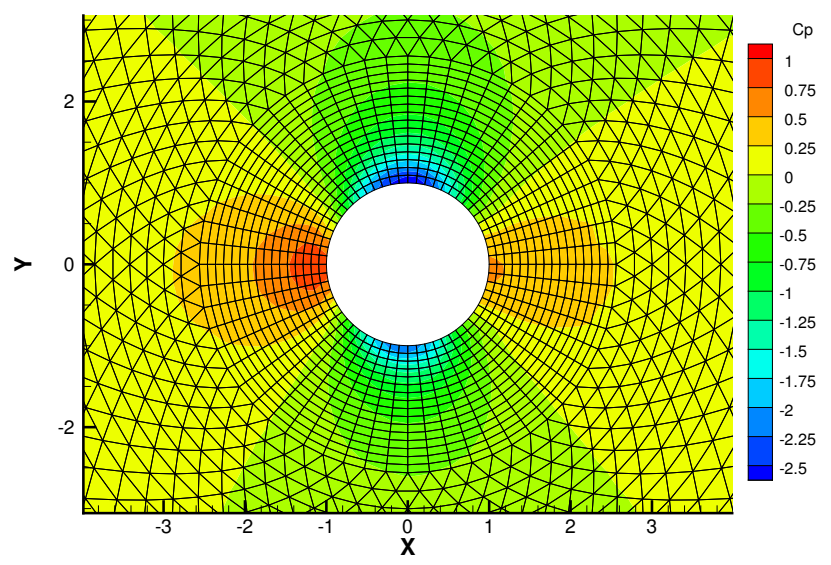

(a) No Modification

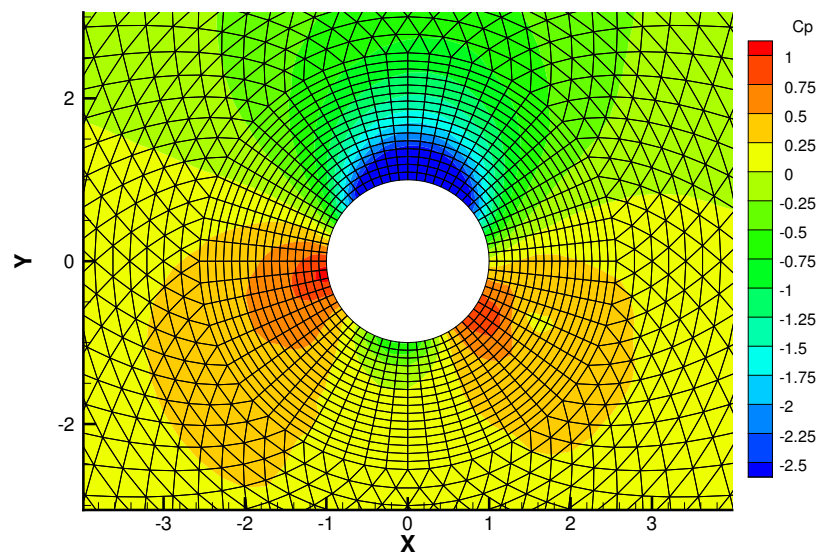

(b) Low Mach-number Modification of normal and tangential velocity components

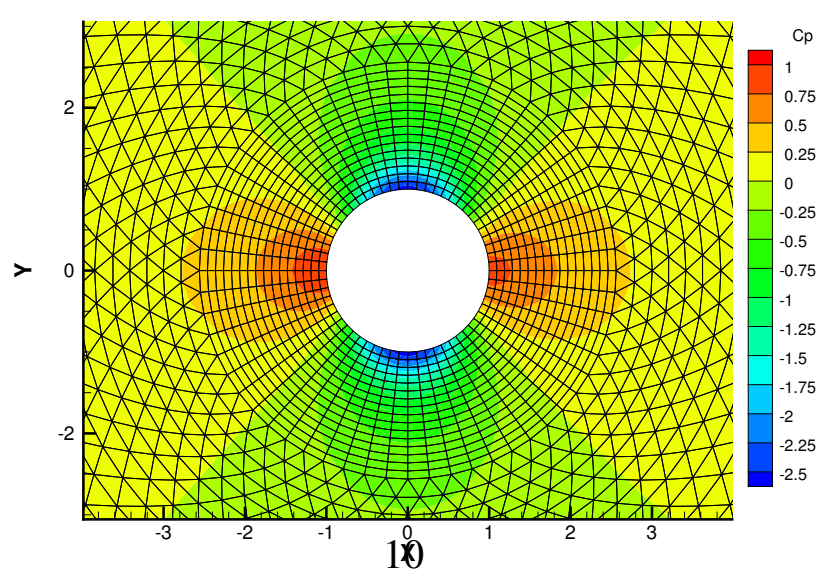

(c) Low Mach-number Modification of normal velocity component

Figure 2: Pressure Coefficient plots using a WENO 3rd order scheme for the flow past a $2 \mathrm{D}$ cylinder at $t=200 \mathrm{~s}$ 


\subsection{D Low Mach number past a Cylinder}

Examined is the steady-inviscid problem of flow past a circular cylinder with radius $\mathrm{R}=1$. This is cut out of a cylindrical computational domain of radius $\mathrm{R}=20$, with a freestream inflow of Mach number $M_{\infty}=0.1$, density $\rho_{\infty}=1$ and the pressure $p_{\infty}=\frac{\rho_{\infty}}{\gamma}$, where $\gamma=1.4$. The flow is initialised with a flow direction parallel to the $\mathrm{x}$-axis.

The simulations were undertaken on two different mesh topologies, a completely triangular mesh with a linear piecewise construction for the cylinder and a hybrid mesh with prism cells near the wall of the cylinder before moving to triangular cells. The computations were ran until $t=200 s$ when the initial transients would have vanished and the residuals would have converged.

The numerical simulations on the fully triangular mesh did not suffer the same dissipation that can be found in the hybrid mesh. Presented in Fig. 2 are the hybrid mesh and pressure coefficient $C_{p}$ distribution on a medium grid of containing 960 quadrilateral and 3316 triangular elements, computed using a WENO 3 reconstruction scheme. It can be seen that both the simulation without any treatment and the simulation using the second treatment suffered dissipation and did not exhibit the symmetry expected from this case. The presence of the first low-mach modification gives a much more accurate solution, with a clear symettry about $x=0$.

\subsection{D Cylindrical Explosion}

The presented case of an explosion in two dimension is outlined by Toro [35] as a classical Riemann solver problem. Contained within a square domain $[0,2] \times[0,2]$, exists a circle of radius $r=0.4$ centered at $(1,1)$, whose internal initial conditions vary from it's external initial conditions.

$$
(\rho, P)=\left\{\begin{array}{cc}
(0.125,0.1) & r>0.4 \\
(1,1) & r \leq 0.4
\end{array}, u=v=0, r^{2}=x^{2}+y^{2}\right.
$$

The solution is the result of a circular shock occurring from the Riemann problem which propagates outwards from the centre, this is followed by a circular contact discontinuity that propagates in the same direction. This is turns leads to a circular rarefaction travelling in the opposite direction to the aforementioned flow features. Over time the shock wave and contact discontinuity will become weaker, resulting in the contact discontinuity to stop and travel inwards while the rarefaction wave with reflect when it reaches the centre.

The solution presented in Fig. 3 was obtained after running the solution until $t=0.2$. A reference solution for density was computer by solving the $1 \mathrm{D}$ problem on a very fine grid. The results indicate that the solver is capable of resolving the shock within 2-3 cells on the unstructured grid and 3-4 on the structured grid, without the need for a lowmach correction. The results using the WENO 5 reconstruction demonstrate that both low-mach corrections can be implemented without inducing any other numerical errors. 


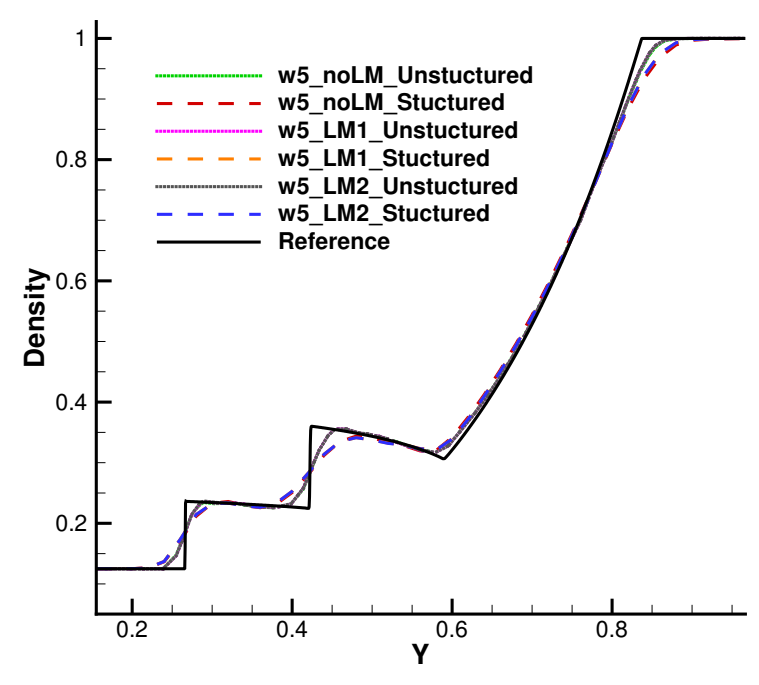

Figure 3: Density plots using Weno 5 numerical scheme with and without the low-mach corrections on both structured and unstructured grids.

\section{Conclusions}

The conclusion drawn from the subject study are listed below:

- A low-Mach number correction to the reconstruction of finite-volume numerical schemes improves the dissipation characteristics of the schemes

- A direct implementation of the original low-Mach number modification of [29] can not be used for all types of elements since they have different dissipation characteristics

- A revised modification is presented since the reconstructed tangential velocity components require more dissipation than the normal velocity components with respect to the interface between two cells

- The subject modification is quite robust to be utilised for all Mach number flows since it is not producing any spurious oscillations at regions of high-Mach number that can contaminate the solution.

\section{Acknowledgments}

The authors would like to thank Jaguar Land Rover for granting permission to publish this work. The work has been undertaken due to funding from EPSRC Award. Ref. Number: 13794. 


\section{REFERENCES}

[1] M. Dumbser, M. Kaser, V. Titarev, E. Toro, Quadrature-free non-oscillatory finite volume schemes on unstructured meshes for nonlinear hyperbolic systems, Journal of Computational Physics 226 (1) (2007) 204-243.

[2] M. Dumbser, D. Balsara, E. Toro, C.-D. Munz, A unified framework for the construction of one-step finite volume and discontinuous galerkin schemes on unstructured meshes, Journal of Computational Physics 227 (18) (2008) 8209-8253.

[3] A. Haselbacher, A weno reconstruction algorithm for unstructured grids based on explicit stencil construction, 2005, pp. 3369-3378.

[4] W. Li, Y. Ren, Quadrature-free non-oscillation finite volume scheme for solving navier-stokes equations on unstructured grids, Vol. 1376, 2011, pp. 639-641.

[5] X. Nogueira, L. Cueto-Felgueroso, I. Colominas, F. Navarrina, M. Casteleiro, A new shock-capturing technique based on moving least squares for higher-order numerical schemes on unstructured grids, Computer Methods in Applied Mechanics and Engineering 199 (37-40) (2010) 2544-2558.

[6] C. Ollivier-Gooch, Quasi-eno schemes for unstructured meshes based on unlimited data-dependent least-squares reconstruction, Journal of Computational Physics 133 (1) (1997) 6-17.

[7] C. O. Gooch, M. V. Altena, A high-order-accurate unstructured mesh finite-volume scheme for the advection-diffusion equation, Journal of Computational Physics 181 (2) (2002) 729-752.

[8] P. Tsoutsanis, V. Titarev, D. Drikakis, Weno schemes on arbitrary mixed-element unstructured meshes in three space dimensions, Journal of Computational Physics 230 (4) (2011) 1585-1601.

[9] V. Titarev, P. Tsoutsanis, D. Drikakis, Weno schemes for mixed-element unstructured meshes, Communications in Computational Physics 8 (3) (2010) 585-609.

[10] W. Wolf, J. Azevedo, High-order eno and weno schemes for unstructured grids, International Journal for Numerical Methods in Fluids 55 (10) (2007) 917-943.

[11] Z. Xu, Y. Liu, C.-W. Shu, Hierarchical reconstruction for discontinuous galerkin methods on unstructured grids with a weno-type linear reconstruction and partial neighboring cells, Journal of Computational Physics 228 (6) (2009) 2194-2212.

[12] Z. Xu, Y. Liu, C.-W. Shu, Hierarchical reconstruction for spectral volume method on unstructured grids, Journal of Computational Physics 228 (16) (2009) 57875802 . 
[13] P. Tsoutsanis, A. Antoniadis, D. Drikakis, Weno schemes on arbitrary unstructured meshes for laminar, transitional and turbulent flows, Journal of Computational Physics 256 (2014) 254-276.

[14] B. Cockburn, C.-W. Shu, Runge-kutta discontinuous galerkin methods for convection-dominated problems, Journal of Scientific Computing 16 (3) (2001) 173-261.

[15] J. Dennis, R. Nair, H. Tufo, M. Levy, T. Voran, Development of a scalable global discontinuous galerkin atmospheric model, Int. J. Comp. Sci. Eng.

[16] A. Uranga, P.-O. Persson, M. Drela, J. Peraire, Implicit large eddy simulation of transition to turbulence at low reynolds numbers using a discontinuous galerkin method, International Journal for Numerical Methods in Engineering 87 (1-5) (2011) 232-261.

[17] J. Zhu, J. Qiu, C.-W. Shu, M. Dumbser, Runge-kutta discontinuous galerkin method using weno limiters ii: Unstructured meshes, Journal of Computational Physics 227 (9) (2008) 4330-4353.

[18] Z. Wang, Spectral (finite) volume method for conservation laws on unstructured grids. basic formulation, Journal of Computational Physics 178 (1) (2002) 210 251.

[19] Z. Wang, Y. Liu, Spectral (finite) volume method for conservation laws on unstructured grids. ii. extension to two-dimensional scalar equation, Journal of Computational Physics 179 (2) (2002) 665-697.

[20] Z. Wang, L. Zhang, Y. Liu, Spectral (finite) volume method for conservation laws on unstructured grids iv: Extension to two-dimensional systems, Journal of Computational Physics 194 (2) (2004) 716-741.

[21] Y. Zhou, Z. Wang, Implicit large eddy simulation of transitional flow over a sd7003 wing using high-order spectral difference method, 2010.

[22] C. Breviglieri, A. Maximiliano, E. Basso, J. Azevedo, Improved high-order spectral finite volume method implementation for aerodynamic flows, 2009.

[23] R. Abgrall, On essentially non-oscillatory schemes on unstructured meshes: analysis and implementation, Journal of Computational Physics 114 (1) (1994) 45-58.

[24] D. Stanescu, W. Habashi, Essentially nonoscillatory euler solutions on unstructured meshes using extrapolation, AIAA Journal 36 (8) (1998) 1413-1416. 
[25] G. S. Jiang, C. W. Shu, Efficient implementation of weighted eno schemes, Journal of Computational Physics 126 (1) (1996) 202-228.

[26] J. Shi, C. Hu, C.-W. Shu, A technique of treating negative weights in weno schemes, Journal of Computational Physics 175 (1) (2002) 108-127.

[27] C.-W. Shu, S. Osher, Efficient implementation of essentially non-oscillatory shock-capturing schemes, Journal of Computational Physics 77 (2) (1988) 439471.

[28] O. Friedrich, Weighted essentially non-oscillatory schemes for the interpolation of mean values on unstructured grids, Journal of Computational Physics 144 (1) (1998) 194-212.

[29] B. Thornber, A. Mosedale, D. Drikakis, D. Youngs, R. Williams, An improved reconstruction method for compressible flows with low mach number features, Journal of Computational Physics 227 (10) (2008) 4873-4894.

[30] P. Tsoutsanis, I. Kokkinakis, L. Konozsy, D. Drikakis, R. Williams, D. Youngs, An investigation of the accuracy and efficiency of structured and unstructured, compressible and incompressible methods for the vortex pairing problem, Computer Methods in Applied Mechanics and Engineering 293 (2015) 207-231.

[31] A. F. Antoniadis, P. Tsoutsanis, D. Drikakis, Numerical accuracy in rans computations of high-lift multi-element airfoil and aircraft configurations, in: AIAA (Ed.), 53rd AIAA Aerospace Sciences Meeting, no. AIAA 2015-0317, Kissimmee, Florida, 2015.

[32] D. Drikakis, A. F. Antoniadis, P. Tsoutsanis, I. Kokkinakis, Z. Rana, Azure: An advanced cfd software suite based on high-resolution and high-order methods, in: AIAA (Ed.), 53rd AIAA Aerospace Sciences Meeting, no. AIAA 2015-0813, Kissimmee, Florida, 2015.

[33] A. F. Antoniadis, D. Drikakis, I. Kokkinakis, P. Tsoutsanis, Z. Rana, High-order methods for hypersonic shock wave turbulent boundary layer interaction flow, in: AIAA (Ed.), 20th AIAA International Space Planes and Hypersonic Systems and Technologies Conference, no. AIAA 2015-3524, Glasgow, Scotland, 2015.

[34] M. Dumbser, M. Käser, V. A. Titarev, E. F. Toro, Quadrature-free non-oscillatory finite volume schemes on unstructured meshes for nonlinear hyperbolic systems, Journal of Computational Physics 226 (1) (2007) 204-243.

[35] E. F. Toro, Riemann Solvers and Numerical Methods for Fluid Dynamics - A Practical Introduction, Berlin: Springer-Verlag, 1997. 
[36] S. Gottlieb, On high order strong stability preserving runge-kutta and multi step discretizations, Journal of Scientific Computing 25 (112) (2005) 105-128.

[37] C. Hu, C. W. Shu, Weighted essentially non-oscillatory schemes on triangular meshes, Journal of Computational Physics 150 (1) (1999) 97-127. 


\section{Implementation of a low-mach number modification for high-order finite-volume schemes for arbitrary hybrid unstructured meshes}

Simmonds, N.

European Congress on Computational Methods in Applied Sciences and Engineering

Simmons, N., Tsoutsanis, P., Gaylard, A. (2016) Implementation of a low-mach number modification for high-order finite-volume schemes for arbitrary hybrid unstructured meshes, Proceedings of the ECCOMAS Congress 2016 VII European Congress on Computational Methods in Applied Sciences and Engineering, 5 - 10th June 2016, Crete, Greece https://www.eccomas2016.org/proceedings/pdf/8545.pdf Downloaded from Cranfield Library Services E-Repository 